\title{
Planning from Within and Alongside Informal Urban Metabolism: A Multi-agent Approach to Adaptive Masterplanning
}

\author{
TREVOR RYAN PATT
}

Singapore University of Technology and Design

\begin{abstract}
This paper describes a possible adaptive approach to redevelopment planning in Guangzhou's urban villages that taps into the material processes of an existing metabolism. Rather than attempting to control an entire plan, this approach sets in motion a continuous swarm of random walks within a multiagent simulation, each agent analysing partial fragments of the urban fabric as they move. The various analyses of the agents are aggregated and form a fuzzy picture of the current situation from which small, punctual interventions (new pedestrian passageways, small public spaces, etc.) can be proposed. Such proposals are paired with reconstruction methods already occurring throughout the village in order not to disrupt the unique character of the urban fabric and to allow the model to engage with the ongoing sociomaterial practices within the village. Prominent among these is a cycle of recycling material from demolished buildings into new structures. This approach differs from existing strategies that cannot incorporate informal practices and rely on representational tropes or importation of entirely new plans based on a complete demolition.
\end{abstract}

The redundancy of the multiagent system and its continuous evolution make it robust to aberrations and therefore capable of adapting to unplanned or external changes on the fly, which allows informal constructions to continue alongside the model without issue. The results of this model suggest that small adaptations, effected through discrete and uncoordinated operations, can be an effective route to redevelopment that does not require a displacement of informal activities but rather follows their direction.

The city of pedestrian acts is one expressed through material action. According to Michel de Certeau, it is a city "shaped out of fragments of trajectories and alterations of spaces." ${ }^{1}$ This is a city whose identity is composed of encounters between people walking around with no coordinated plan, but also from the encounters these pedestrians have with the built environment-the buildings and spaces that shape their movement or provide shelter-as well as the encounters across material objects themselves. The leaky roof that gradually gives way to decay, neighboring buildings that splice off of the same power line, tall structures that block out the sun and GPS signals: these sociomaterial interactions are also at work rewriting the city in shifting and unexpected ways. In contrast, de Certeau calls the city of space planner urbanists, city planners, and cartographers a "geometric" space that is out of time or place, a panoptic and synchronic system that maintains only an optical relationship to its object, entirely disentangled from such material realities of the city.

There is no suggestion that these two cities are mutually exclusive, both frequently overlap in the same locations, but particularly in informal urban settlements we can see more clearly and more fully how the pedestrian city occurs and the resistance that it poses to the geometrical city's attempts to identify, represent, and plan for it. These struggles are exemplified in China's "urban village," a phenomenon that occurs when once-rural land is engulfed by the rapid expansion of a nearby megalopolis. ${ }^{2}$ Because China maintains a strict regulatory demarcation between collectively-owned 'rural' lands, and state-owned 'urban' classifications, it is bureaucratically complicated to transfer an area's status from one to the other. As a result, it quickly becomes impractical for agricultural production to continue on the rural land and the village converts to rental housing, often becoming reaching the higher population densities than their surrounding cities-Shipai village in Guangzhou, for example, has a population density over $50,000 / \mathrm{km}^{2}$. However, because they remain, in official terms, rural, the development of urban villages occurs outside the purview of planning authorities and does not have access to the full suite of urban infrastructural benefits.

\section{INCOMPATABILITY OF PLANNING APPROACHES TO THE INFORMALITY OF URBAN VILLAGES}

Having withheld political representation from urban villages, it is difficult for the city to regain an understanding of the villages by representational means. With no coordinating planning authority, it is not infrequent for villages to transform faster than they can be surveyed. ${ }^{3}$ In the cases when villages are eventually converted from rural to urban status, the plan most involves a complete demolition of the village and a new reconstruction from scratch. In fact, at one point, the municipal government of Guangzhou had a plan to remove all 138 urban villages in the city by 2015. ${ }^{4}$ The tabula rasa strategy accomplishes a reset to a knowable and manageable condition. Although there have been cases where villagers have managed to negotiate an allocation for themselves within the new developments, prominently at Liede Village adjacent to Zhujiang New Town in Guangzhou, ${ }^{5}$ this still resulted in the village's complete demolition. 


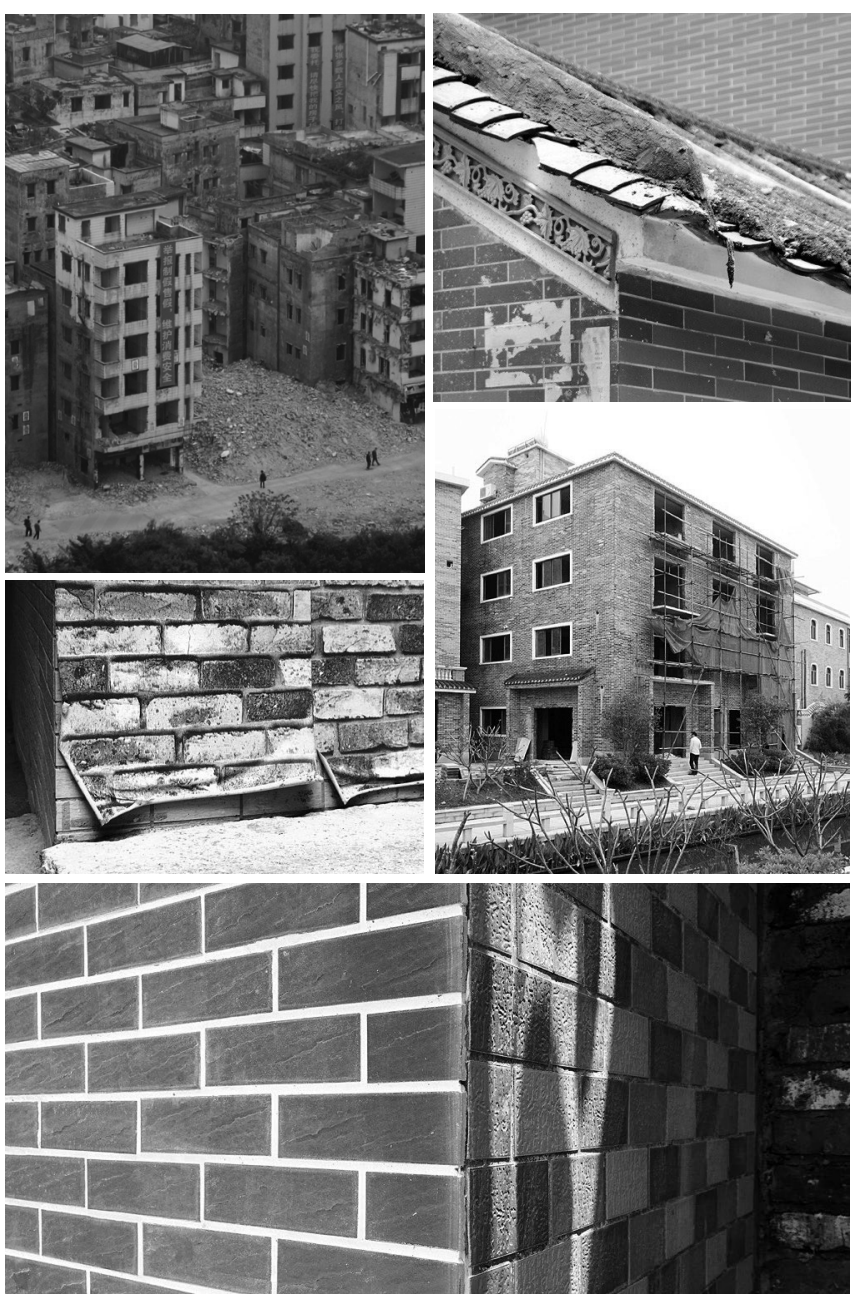

Figure 1: Demolition of an entire urban village adjacent to Zhujiang New Town and reconstruction through simulacra of Lignan style architecture (with various degrees of convincingness) across urban villages in Guangzhou. Prefab plaster mouldings stand in for stone eaves, lintels, and window frames while brickwork is tpyically represented with a veneer of tiles-but in at least one occasion by sheets of paper. Often this fancier grey tile is only applied to the front façade.

A less drastic approach was taken at Huangpu Village. This village was an historic port and home to a number of Lingnan-style heritage sites. Here, a partial clearing was done to create a pedestrian promenade for tourists to circulate. The remainder of the village was treated to a 'beautification' project that consisted primarily of refacing the existing buildings that faced this promenade with a combination of grey tile veneer and precast concrete details meant to resemble brick and stone Lingnan construction from a distance. From the maps meant to keep visitors from wandering off path, to the vigorous sign-posting, and the obvious material simulacra, everything about this exercise underscores how much the 'geometric' city is reliant on visual operations.

Not far away on Haizhu island, there is a series of villages that embody the opposite path of development. These villages are buffered from the most extreme redevelopment pressures by a green belt of former orchards. ${ }^{6}$ Here there were small pushes for beautification but no sustained campaigns; the primary development motivation is coming from within through informal mechanisms. These developments tend to follow a pattern where low residences of one or two stories, often dilapidated are taken down. These houses tend to be brick cavity walls constructions. The bricks are cleaned of mortar, collected and stacked somewhere nearby or around the edge of the village. On the cleared site, new foundations will be dug and poured and a concrete frame (as tall as 8 stories) erected. The narrowness of the pathways through the city means that concrete has to be ported in from the village perimeter by hand in wheelbarrows, or when possible, pumped in via very long pipelines. The frame is infilled with bricks, sometimes new or sometimes recycled from another building recently brought down. Because these infill walls only require a single layer, the same material is now able to cover more surface area. Finally, the new building is covered with small tiles to protect against the rain and the elements.

Apart from the canals and some very narrow alleyways, the built footprint of the village has almost no extant vacant space. This means that before any new building can go up another first needs to come down. At any given moment, dozens of sites across the village are enacting some stage of this cycle from rubble to new midrise apartment. Although there are moments still where a tile veneer is passed off as masonry these villages do not practice a representational urbanism-even signage is sparse-and a fruit market, or a barber shop, or a recreation center exists by virtue of its taking place rather than because it has an assigned a point in the city.

\section{PROPOSING AN ALTERNATE APPROACH}

Inasmuch as this urban metabolism is churning along without external prompting from planners or architects it is unnecessary to problematise a new process of 'inventive' reuse for the urban village. Nor do we feel it is especially urgent to generate new model forms or building types. Rather, our interest is to develop an approach which allows planning concerns to be insinuated within and alongside the existing construction processes. In order to allow the existing metabolism to continue to flourish, they cannot be restricted within the limits of a pre-defined masterplan nor sidelined as merely a secondary process. This means that our approach must be adaptive and contingent upon the informal developments, it should be open-ended rather than deterministic, it should be a dynamic model that is continuously revised, and it needs to work in a distributed way through local adaptations rather than totalizing plans. Approaches like this have not been tried previously because this sort of complexity is exactly the kind that 'geometric' planning would like to avoid, however, advanced computational modelling can adapt to these conditions and manage large sets of changing, contradictory, distributed, and contingent data. 
Following these criteria, this project takes the urban network as the material on which it operates. By addressing dead ends and disjunctions in the public space and circulation network the model can effect greater connectivity and integration of the village fabric. The existing network of streets and alleyways was redrawn and formatted by their topological relationships into a mesh. ${ }^{7}$ Additionally, we included in this mesh the parcel boundaries in order to know where construction processes were likely to start and end. There is a great deal of interesting morphological detail to the inherent structure of the network that can be drawn out using network analysis methods. By overlaying a large set of shortest-path calculations between various points in the village, certain circulation corridors emerge that are evidently more hierarchically important to linking together or crossing the village despite the fact that such circulation spines are not visually apparent in the form. Similarly, pockets of isolated space can be found by plotting the size of the reach from any given point (that is, how much of the village could be reached from some starting point within, for instance, a $120 \mathrm{~m}$ walk in any direction) and comparing the geodesic distance to all of these points to the straight-line distance. The smaller the reach and the higher the ratio of the geodesic to Euclidean distance, the less integrated the point.

One of the methods utilized in this model was to calculate all the points reachable from a node in the network in within an $80 \mathrm{~m}$ walk and the distance to these points. These values were recalculated using the assumption that one could take shortcuts between existing building along the edge of the parcel

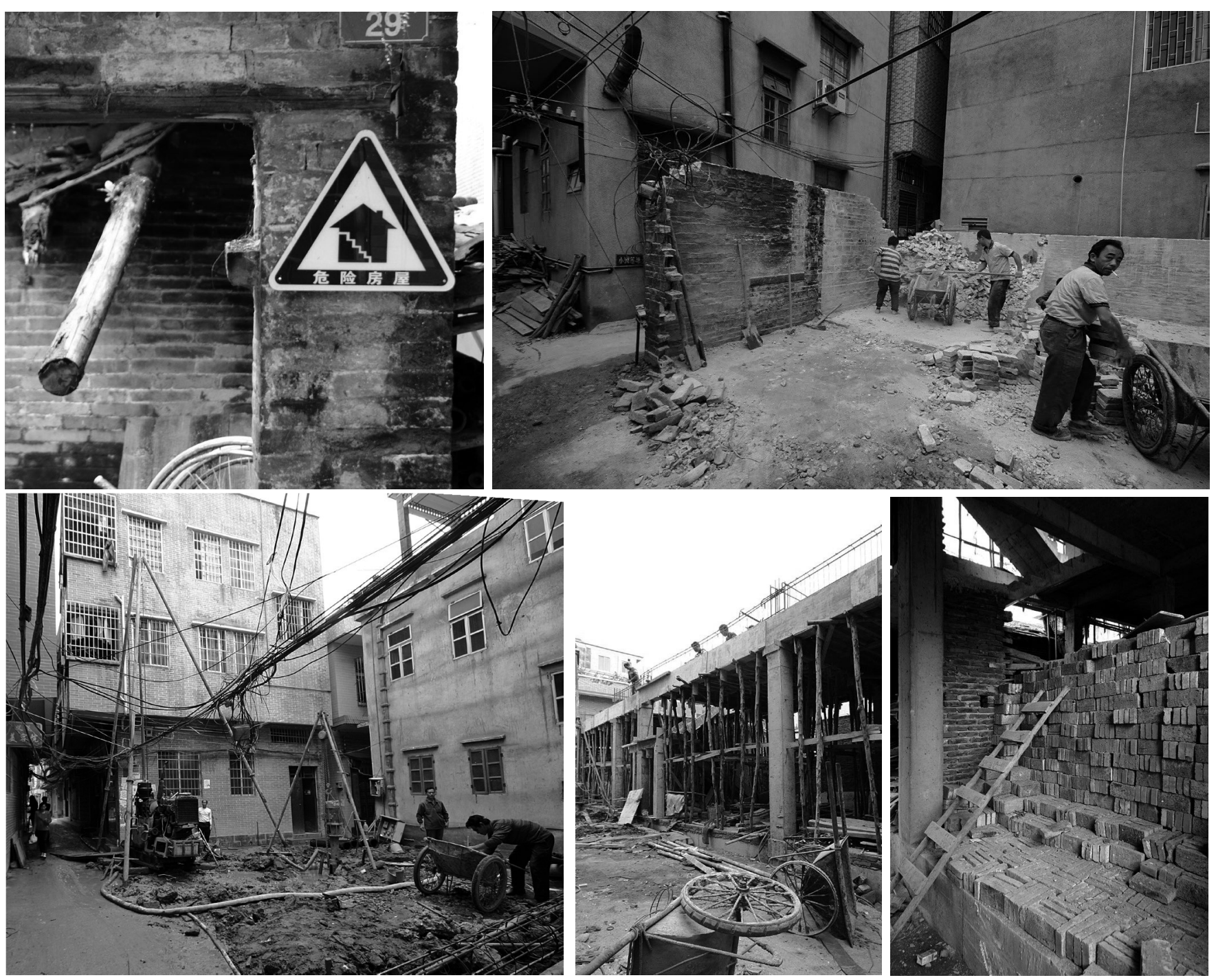

Figure 2: The process of disassembling and salvaging brick from older buildings, pouring new foundations for new, multistory concrete frame structures, and the return of bricks to be fitted in as infill plays out across the village on many different sites. 

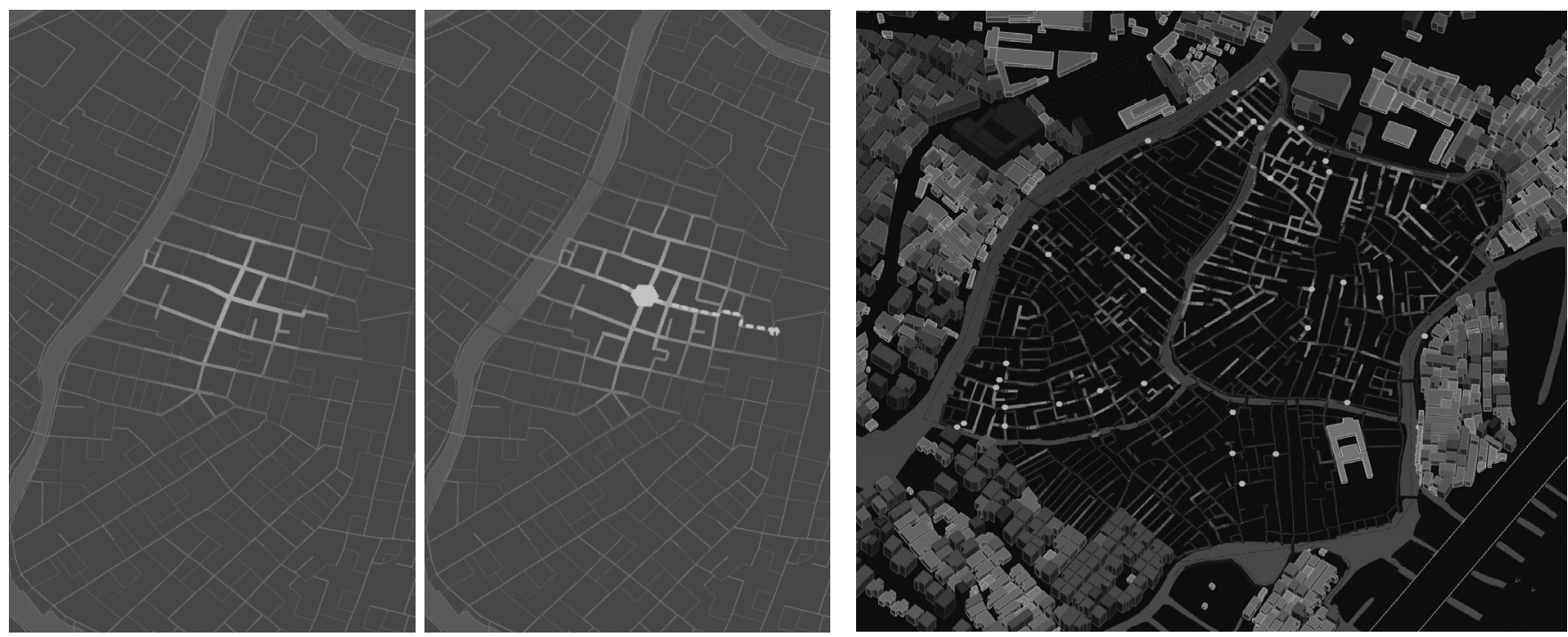

Figure 3: Agent operations within the model. Left: a comparison of a localized network reachl analysis and the identification of a potential shortcut path (in blue) when the network analysis incorporates the plot divisions. Right: an example of accumulated traffic patterns from 40 agents left to run for 500 steps.

boundaries. Identify the most impactful shortcut within this localized network suggested possibilities for new pedestrian pathways that could be proposed should one of the adjacent buildings come down.

The second component that contributes to the model is the simulation of a multiagent system that distributes the analyses across the site and gives the model its dynamism. For this model we have written an agent class that moves in a random walk along the network from node to node. These walks can be a purely random choice of direction at each intersection or they can be directed to certain goals. Here we have given parameters to prevent direct backtracking (except at a dead end) and to give a slight preference to wider streets in order to produce traffic patterns that somewhat mimic pedestrian usage. It is hypothesized that the random walks implicitly reproduce patterns of clustering in the morphological structure of the network. ${ }^{8}$ The traces of the random walks are saved to the links in the network to record traffic levels around each building parcel. At each step in the simulation, every agent also analyzes its local network to locate possible shortcuts as described above.

In the operation of the model a large number of agents (up to 40 at a time) is deployed across the village, starting from randomly selected locations chosen from a list of public spaces and gathering points identified during site visits. As the agents walk over the network, an aging factor gradually phases out each agent (more quickly if they fall into repetitive patterns) and replaces it with a new one at a new location in order to ensure an even coverage. Both the traffic values and the shortcut locations are recorded into the network as the simulation runs. Meanwhile, the building stock is also transforming: an age factor is assigned to each building that decreases over time signifying an increased chance of replacement. The values of the persistence of the building stock is periodically compared to the combined 'demand' of traffic and shortcut values. Parcels whose persistence value is surpassed by the sum of the demand around their perimeter are identified as likely to need replacement. In our study, one of these potential sites is selected at random, in reality this step would be replaced by a discussion with the property's manager and the village committee.

The model takes advantage of this moment of reconstruction to introduce small, punctual adaptation of the circulation network, adding in the shortcut links by making small offsets to the building perimeter at the first stories of the new building. This follows the existing ubiquitous 'handshake building' typology-ubiquitous in Chinese urban villages - that extends out over the pedestrian alleyways to nearly meet its neighbour in the middle. The new link is also acknowledged in the mesh datastructure so that it will be treated as a traversable path as the simulation continues and will be counted as such in the network analyses. After the new pathways are established, the new building is also projected back into the model. Acknowledging that the motivation for reconstruction is typically to increase the rentable space, the new structure is assigned a greater height than the building it replaces. We calculate the new volume by factoring the total surface area that could be enclosed by reassembling the bricks of the original structure (assuming a masonry cavity wall construction) as infill panels within a concrete frame structure.

As a test case, the simulation was run for a duration of 5000 steps. In this duration the overall floor area ratio of the village nearly doubled from an initial value of 1.27 to 2.48 . Approximately one-third (32.3\%) of the buildings in the village were affected with $70,000 \mathrm{~m}^{2}$ of floor area renovated and $180,000 \mathrm{~m}^{2}$ of new area projected. Additionally, 62 new pocket 
plazas were opened up and 653 new shortcut passageways. The average length of a new pathway was less than 7 meters and these interventions were scattered all across the village. To assess the impact of the modifications, we calculated the eccentricity at each node in the network (that is, the distance required to get to the farthest away point where lower values are more central and higher values more eccentric) before and after the simulation was run. Both the median and maximum eccentricity values dropped by around $10 \%$ from $565.5 \mathrm{~m}$ and $775.3 \mathrm{~m}$ to 508.7 and $702.1 \mathrm{~m}$ respectively. This demonstrates that the model capable of addressing the qualities of the urban form as a macro assemblage, despite only operating on small elements and without a panoptic understanding of the whole, but only dispersed and localized analyses.

Although our case study was run as a self-contained simulation it is possible to pause the model's operation and to input or adjust the data in the model partway through. In a real application, this would allow the initial data to be updated as more detailed surveying was carried out or as new bottom-up redevelopment occurred separate from the model's recommendations. One could also imagine the model being paused at each round of new changes while the planners consulted with the villagers on the various proposals generated at that stage. In this way, the model can become a tool for discussion and negotiation between different parties that respects the self-determination of the village and not a prescriptive plan. If any of the proposals are accepted, they would be confirmed in the model, if not, the simulation can be continued for another round with no change. The myopia of the agents in only being able to see or operate on a small, localized bit of the network at any time makes the model robust to the occasional inaccuracy in a way that a totalizing or optimized approach would not be. Finally, one last advantage of this model is that, like the urban village, the simulation is inherently entropic. Unlike many dynamic planning models that ultimately reach an end state of equilibrium, this model will continue to operate endlessly unless it is somehow externally starved of material.

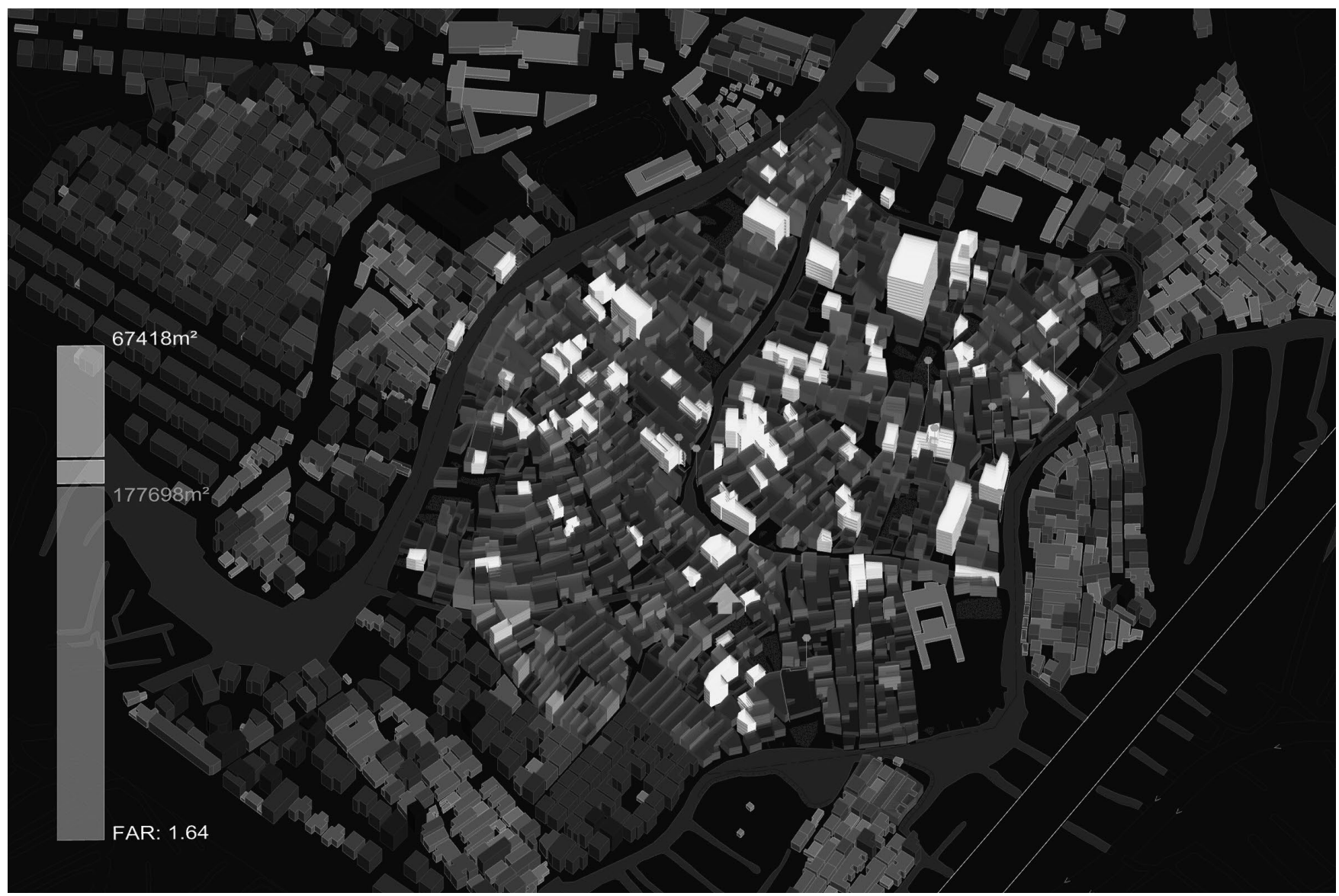

Figure 4: A still frame from the simulation illustrating how the visual interface to the model appears during operation. The grey tone of each building mass indicates its aging factor. White volumes denote buildings that have been reconstructed and yellow volumes are their new extensions. An up-to-date sum of the floor areas is displayed by the bar graph on the left of the screen using the same color code. Buildings that are in the process of demoltion or reconstruction are marked with a red down arrow or a yellow up arrow. An alternate view of visualization shows only the building footprints, allowing the agent movement and traffic values of the network to be read more clearly. 


\section{CONCLUSION}

The aim of the project is to bridge the separation between the domain of planning and the realm of material enaction by demonstrating a new model for planners to engage with urban informality. At present, the largest barrier to this is simply that conventional planning methods are incapable of handling so much complexity and variability. It is necessary to create tools that provide space for a moment of interaction between the formal and informal. However, the more fundamental barrier is that in order not to extinguish informal activity, planning must also change the object on which it operates and the material with which it works. In this example, the object of the model is to make it possible for an existing material culture to participate in the urban and to address the production of public space for a common benefit.

Following the definition of assemblage as "the capacity to act with the coming together of things," ${ }^{\prime \prime}$ the Haizhu villages are significant because they ultimately reveal themselves as the product of bringing together a truly unique set of sociomaterial circumstances. The metabolic activity of the villages shows us how an adaptive and opportunistic reclamation of waste material might actually play out at a broad scale, ${ }^{10}$ and it is through this example-as a real phenomenon and not only hypothetical-that we can see the potential to extend to these emerging material practices an agency that was previously reserved for planners, understood solely in a representational mode. This project argues that the productivity of informal practices can, in fact, be instrumentalized without subordinating their action to an external plan, but by dissasembling any notion of comprehensive masterplan, distributing the underlying operations, as though they were also some diffuse raw material, and placing these into the mix, to seek new connections and to flow alongside the site's own trajectories.

\section{ENDNOTES}

1. Michel de Certeau, The Practice of Everyday Life, trans., Steven Rendell (Berkeley: University of California Press, 1984), 93.

2. Him Chung, "Building an Image of Villages-in-the-City: A Clarification of and Regional Research 34, no. 2 (June 2010): 421-437; Katrina Mullan, Pauline Grosjean, and Andreas Kontoleon, "Land Tenure Arrangements and Rural-Urban Migration in China," World Development 39, no. 1 (2011): 123-133; Ya Pevelopment in China: Urban Villages in Shenzhen," International Journal of Urban and Regional Research 33, no. 4 (December 2009): 957-973.

3. Pu Hao, Richard Sliuzas, and Stan Geertman, "Race Against Planning: Unplanned Urban Spaces in Shenzhen," in New Towns for the 21st Century: The Planned vs. the Unplanned City, ed. Michelle Provoost (Amsterdam: SUN, 2010).

4. Margaret Crawford and Jiong Wu, "The Beginning of the End: Planning the Destruction of Guangzhou's Urban Villages," in Villages in the City: A Guide

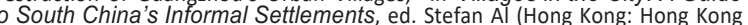
University, 2014), 19-28.

5. Crawford and $\mathrm{Wu}, 19-28$.

6. The orchards have been partially converted into an eco-park. Together, the remaining orchards and the park have been retained in a strategy to serve as the 'green lung' of Guangzhou. Timothy Morton, Hyperobjects (Minneapolis: University of Minnesota Press, 2013).

7. Specifically a half-edge mesh as detailed in Mark de Berg et al., Computational Geometry: Algorithms and Applications (Heidelberg: Springer, 2008).

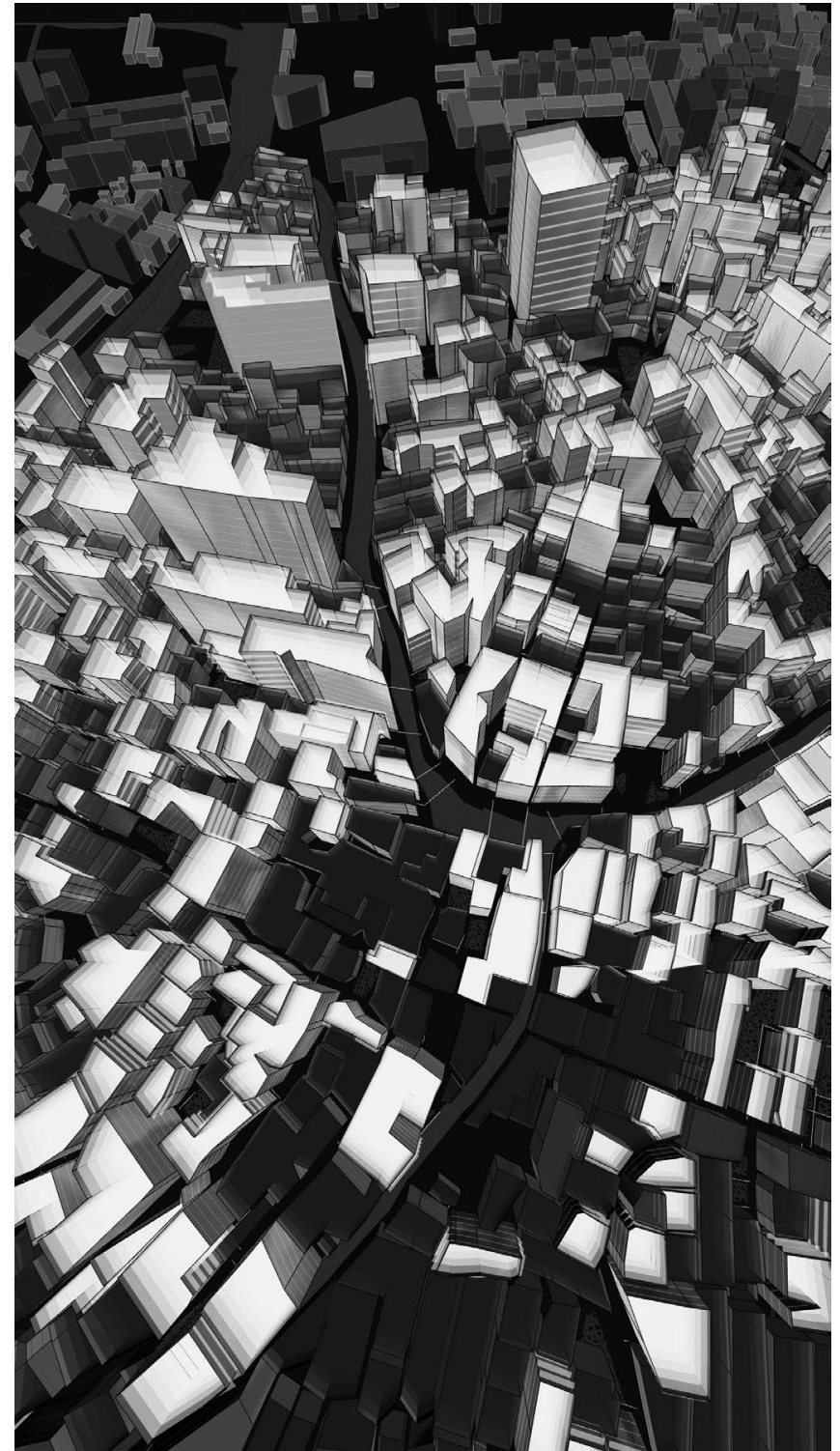

Figure 5: A perspective view of the end result of the simulation showing the extent of transformation after having been run for 5000 steps.

8. Morteza Alamgir and Ulrike von Luxburg, "Multi-Agent Random Walks for Local Clustering on Graphs," in 10th International Conference on Data Mining (Piscataway, NJ: IEEE, 2010), 18-27; David Harel and Yehuda Koren, "On Clustering Using Random Walks," in FCT' TCS 2001: Foundations of Software Technology and Theoretical Computer Science (Bangalore: Springer, 2001), 18-41.

9. Bruce Braun, "Environmental Issues: Inventive Life," Progress in Human Geography 32, no. 5 (2008): 667-679.

10. Compare to the contributions of Nikole Bouchard and Adam Fure, et.al., in this volume. 